\title{
Papillomatosis of the extrahepatic bile ducts and gallbladder diagnosed by endoscopic ultrasound (EUS) and endoscopic retrograde cholangiopan- creatography (ERCP)
}

A 57-year-old man was referred for a diagnostic workup because of elevated serum liver enzyme levels ( $\gamma$-glutamyltransferase $53.94 \mu \mathrm{kat} / \mathrm{L}$, reference range 0.00-0.63). A transabdominal ultrasound revealed dilated extrahepatic bile ducts, and on endoscopic ultrasound (EUS) the common bile duct (CBD) was dilated with hyperechoic foci with no acoustic shadowing within, and a 20-mm hyperechoic frond-like mass was noted within the infundibulum of gallbladder ( $\bullet$ Fig. 1). The CBD and gallbladder walls were thin with smooth outer margins. Endoscopic retrograde cholangiography (ERC) revealed a 15-mm long filling defect within the dilated CBD ( $\bullet$ Fig. 2). A biopsy sample taken from the CBD showed evidence of tubulopapillary adenoma with foci of well-differentiated adenocarcinoma. An abdominal computed tomography (CT) scan showed atypical, but noncontrast-enhancing, hyperdense structures within the gallbladder infundibulum, which were not suggestive of tumor $(\checkmark$ Fig. 3). The patient underwent open laparotomy, cholecystectomy, and extensive resection of the extrahepatic bile ducts with Roux-en-Y hepaticojejunostomy. The histological examination confirmed the diagnosis of papillomatosis of the extrahepatic bile ducts, with foci of high-grade dysplasia ( Fig. 4) and secondary growth extending into the gallbladder along the cystic duct.

Biliary papillomatosis (BP) is a rare disease characterized by the presence of numerous papillary adenomas within the intraand/or extrahepatic biliary tree, with or without mucin hypersecretion [1]. It is a premalignant condition, undergoing malignant transformation in $25-50 \%$ of cases [1]. The diagnosis is based on histological examination of specimens obtained during ERC, cholangioscopy, or surgery. Papillary carcinoma, which manifests as a welldefined, polypoid mass obstructing the lumen of a single extrahepatic bile duct and as a single filling defect on ERC, should be distinguished from multicentric biliary papillomatosis.

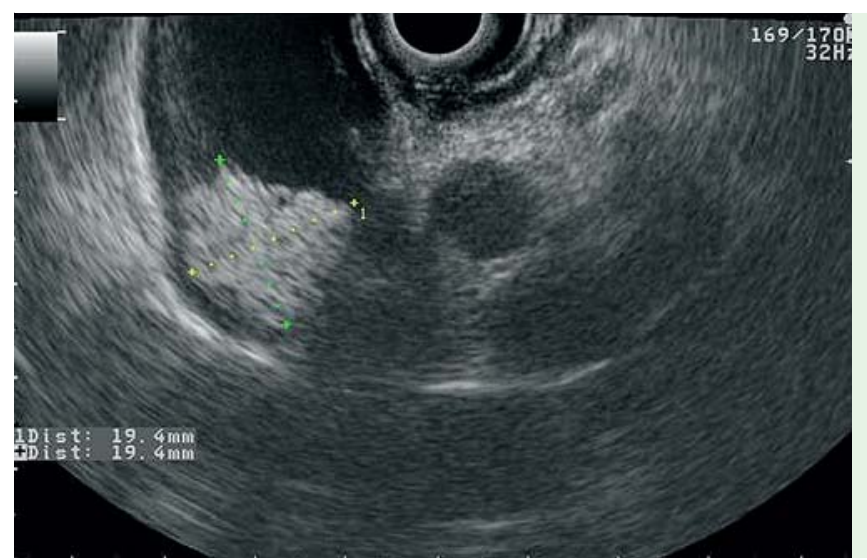

Fig. 1 Hyperechoic, frond-like mass within the gallbladder infundibulum of an older man presenting with elevated liver enzymes.

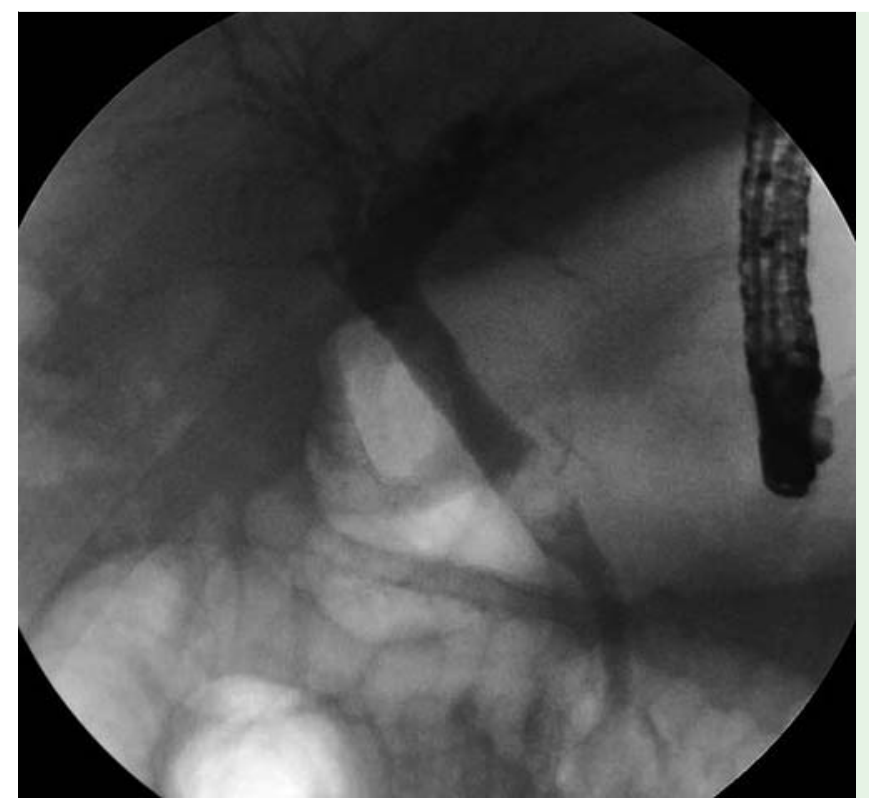

Fig. 2 A filling defect was noted in the dilated common bile duct.

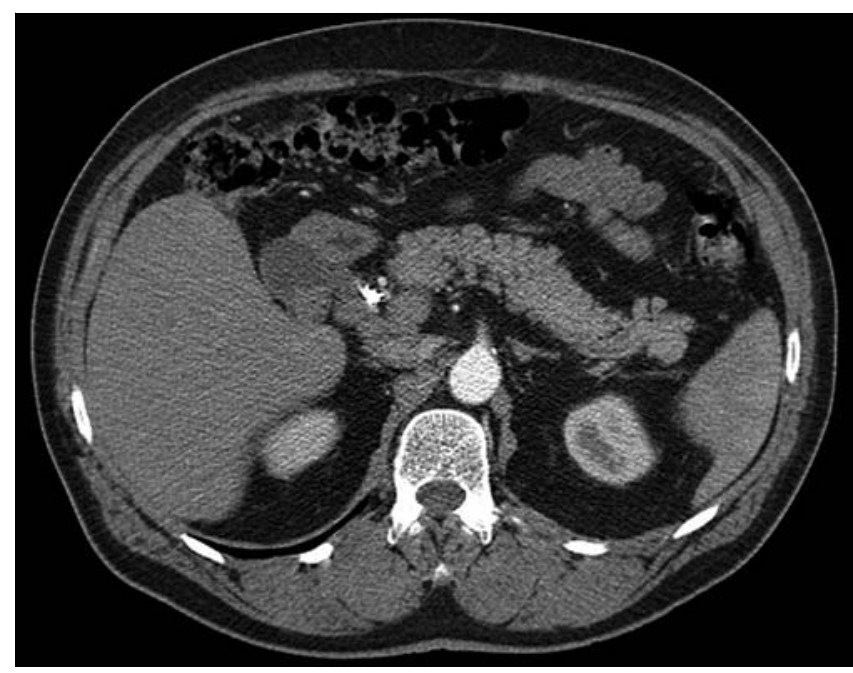

Fig. 3 Atypical dense structures within the gallbladder infundibulum. 


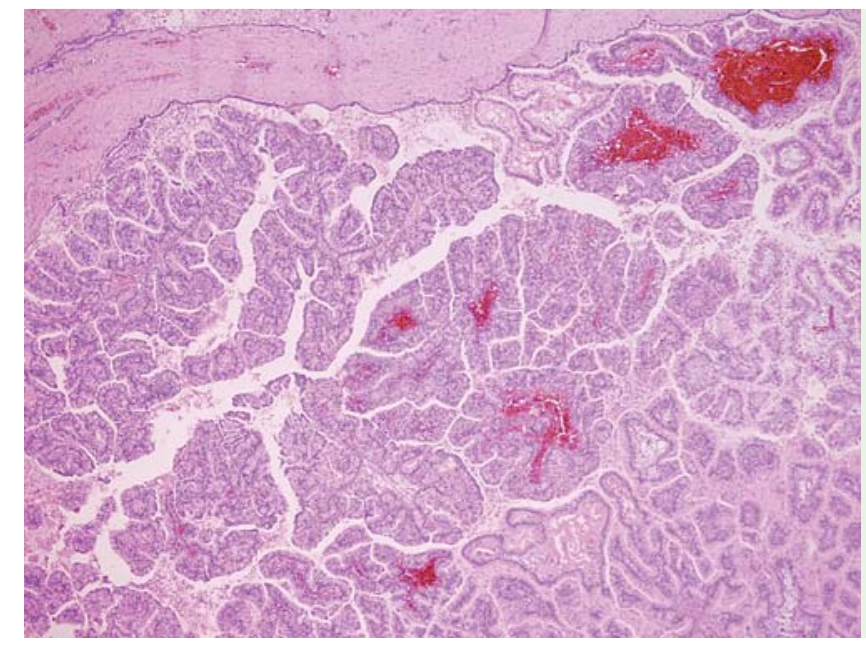

Fig. 4 The dilated common bile duct with papillary clusters of epithelial cells showing high-grade dysplasia (hematoxylin and eosin, magnification $\times 40$ ).
M. Kliment ${ }^{1}$, O. Urban ${ }^{1,2}$, M. Straka ${ }^{3}$, L. Kudelka ${ }^{4}$, P. Fojtik ${ }^{1}$, P. Falt ${ }^{1}$

1 Department of Gastroenterology, Hospital Vitkovice, Ostrava, Czech Republic

2 Faculty of Medicine, University of Ostrava, Czech Republic
3 Department of Surgery, J. G. Mendel Oncology Centre, Novy Jicin, Czech Republic

${ }^{4}$ Department of Pathology, P\&R Laboratory, Novy Jicin, Czech Republic

\section{Reference}

1 Lee SS, Kim MH, Lee SK et al. Clinicopathologic review of 58 patients with biliary papillomatosis. Cancer 2004; 100: 783 - 793

\section{Bibliography}

DOI $10.1055 / \mathrm{s}-0030-1256733$

Endoscopy 2011; 43: E321 -E322

(c) Georg Thieme Verlag KG Stuttgart · New York . ISSN 0013-726X

\section{Corresponding author}

\section{Kliment}

Department of Gastroenterology

Hospital Vitkovice

Zaluzanskeho 1192/15

703-84 Ostrava

Czech Republic

Fax: +420-595-633077

martin.kliment@nemvitkovice.cz 